\title{
Assessment of coronary artery stenosis by magnetic resonance imaging
}

Dudley J Pennell, Hugo G Bogren, Jennifer Keegan, David N Firmin, $S$ Richard Underwood

\begin{abstract}
Objective-The findings of magnetic resonance and $x$-ray angiography were compared for assessment of coronary artery stenosis in this validation study.

Background-Magnetic resonance angiography of the coronary arteries has recently been described, but there has been no comparison with $x$-ray angiography of localisation or assessment of important characteristics of coronary stenosis.

Methods-A breath hold, segmented k-
\end{abstract} space, 2D gradient echo imaging technique incorporating fat suppression was used in 39 patients ( 55 coronary stenoses) with known coronary artery disease.

Results-Overall, 47 stenoses $(85 \%)$ were assessed by magnetic resonance ( 29 of 33 stenoses in the left anterior descending artery, one of one in the left main stem, 14 of 17 in the right coronary artery, and three of four in the left circumflex artery were detected). There was close agreement between magnetic resonance and $x$ ray angiography for the distance of the stenosis from the arterial origin (magnetic resonance mean (SD) 27 (16) $\mathrm{mm}$ versus $x$-ray angiography $27(16) \mathrm{mm}$, $P=N S$, mean difference $-0.2 \mathrm{~mm})$. The distance to 39 stenoses $(83 \%)$ agreed to within $5 \mathrm{~mm}$, with increased scatter for more distal stenoses. The severity of magnetic resonance signal loss, assessed visually at the site of stenosis, varied significantly according to the percentage diameter stenosis $(F=30, P<0.0001)$; stenosis severity with severe signal loss was 89 (7)\%, with partial signal was 70 (16)\%, and with irregular wall only 37 (11)\%, with significant differences among the three groups $(P<0.001)$. A significant correlation was found between the proportional magnetic resonance signal loss at the stenosis and the percentage diameter stenosis severity $(r=-0.67, P<$ $0 \cdot 0001)$. The length of stenosis measured by magnetic resonance (6 (3) $\mathrm{mm}$ ) was greater than by $x$-ray angiography (5 (2) $\mathrm{mm}, P<0.006$, mean difference $+1 \cdot 1$ mm). Spearman's rank test showed that there was significant overestimation of stenosis length by magnetic resonance as stenosis severity increased $\left(r_{\mathrm{s}}=0.34, \mathrm{P}<\right.$ 0.02).

Conclusions-Accurate localisation of coronary stenosis and a qualitative assessment of stenosis severity are possi- ble by magnetic resonance, but stenosis length is overestimated as severity increases, probably because of disturbed patterns of flow with turbulence distal to severe stenoses. Reasonable results for the detection of coronary artery stenosis by magnetic resonance were achieved in this highly selected population, but further progress in imaging techniques is necessary before moving towards appreciable clinical application.

(Heart 1996;75:127-133)

Keywords: coronary artery stenosis; magnetic resonance angiography; $x$-ray angiography

Imaging of the coronary arteries to localise stenosis, measure severity and assess the distal vessel is important in the management of coronary artery disease, and is essential before revascularisation by coronary bypass surgery or angioplasty. The invasive technique developed over the past 35 years, however, uses intra-arterial catheterisation and injections of contrast medium, with significant $x$-ray exposure and a small risk of death and other complications. ${ }^{12}$ At present there is no satisfactory alternative, but magnetic resonance coronary angiography is under development despite formidable problems which include small vessel calibre and tortuosity, cardiac and respiratory motion, and proximity to other tissues of high water density. The magnetic resonance technique which has shown good initial results combines a segmented k-space $2 \mathrm{D}$ gradient echo acquisition with fat suppression within the period of a breath hold, ${ }^{34}$ but there are little published data on its application in coronary artery disease. ${ }^{56}$ The aim of this study was to determine the ability of magnetic resonance imaging to localise coronary stenosis and assess other characteristics such as severity and length.

\section{Patients and methods}

MAGNETIC RESONANCE IMAGING TECHNIQUE Magnetic resonance imaging was performed using a velocity compensated $2 \mathrm{D}$ gradient echo sequence (TE $6.5 \mathrm{~ms}$, TR $15.7 \mathrm{~ms}$ ) and a segmented k-space technique, ${ }^{7}$ such that eight phase encoding steps were acquired in each cardiac cycle. Images of $128 \times 256$ matrix were acquired over 16 cardiac cycles during breath holding at end expiration. No respiratory feedback was used. The acquisition 
window for the eight phase encoding steps was $126 \mathrm{~ms}$ placed in mid to late diastole, during the period of minimal cardiac motion. Because of the high signal from fat which surrounds the coronary arteries, fat signal suppression was employed, using frequency selective preexcitation and dephasing before water excitation. Studies were performed using a $1.5 \mathrm{~T}$ system (Picker International Vista scanner, Cleveland Heights, Ohio, USA), with a $60 \mathrm{~cm}$ patient bore. At this field strength, the fat frequency was approximately $210 \mathrm{~Hz}$ higher than water. The field of view was $20 \mathrm{~cm}$ and the slice thickness $5 \mathrm{~mm}$, giving an in plane pixel size of $1.6 \times 0.8 \mathrm{~mm}$. The images were interpolated to $512 \times 512$ for display. Imaging was performed in the supine position using a lumbar spine surface coil over the anterior chest. The duration of imaging was about $1 \mathrm{~h}$.

\section{CHARACTERISTICS OF PATIENTS}

We studied 39 patients ( 36 men) with chronic stable angina, who had a mean (SD) age of 55 (9) years. All patients were considered capable of cooperating with breath holding and were in sinus rhythm. All were undergoing clinical investigation for chest pain, and at the time of magnetic resonance imaging were known to have either an abnormal thallium tomogram or $x$-ray coronary angiogram. The abnormal perfusion territory or artery was used as a guide for which artery to image by magnetic resonance to obviate imaging of normal arteries. Other than this, no further details of the artery were known at the time of magnetic resonance imaging. All patients underwent $x$-ray coronary angiography within $1 \cdot 2(2 \cdot 4)$ months and there were no significant clinical events between magnetic resonance imaging and $x$ ray coronary angiography. There were 55 stenoses with a luminal diameter narrowing of greater or equal to $50 \%$, of which 33 were located in the left anterior descending artery (17 proximal third and 16 mid-third), 17 in the right coronary artery (eight proximal third, eight mid-third, and one in the posterior descending artery), four in the left circumflex artery (all proximal third), and one in the left main stem.

\section{IMAGING PLANES USED TO IDENTIFY THE} CORONARY VESSELS

Transaxial images at the level of the coronary sinuses were acquired to show the origins of the coronary arteries. Contiguous overlapping slices were then acquired in the transaxial plane to show the course of the proximal arteries before descent in the atrioventricular sulci or interventricular groove. Longitudinal imaging of the arteries was then performed using multiple contiguous oblique slices with a 4 $\mathrm{mm}$ overlap to ensure complete imaging of the artery as it passed in and out of plane. ${ }^{4}$ The right coronary artery was imaged using oblique planes passing through its origin and its position in the atrioventricular groove. The distal right coronary artery was imaged by locating the artery between the diaphragm and the inferior myocardium with an oblique transaxial image. Imaging was also performed in an oblique sagittal or coronal plane. For the left coronary artery, the main stem was followed to its division with transaxial images. The course of the first part of the left anterior descending artery passing between the left ventricle and the pulmonary artery was imaged in an oblique coronal plane. This showed the proximal artery, the origin of the left circumflex artery, and the downward inclination of this portion. From this, an oblique transaxial image was acquired of the proximal artery. This improved visualisation of the continuation of the proximal portion of the artery passing around the pulmonary artery and included the diagonal branches. Lower transaxial imaging showed the left anterior descending artery in the interventricular groove, and longitudinal oblique imaging again showed the more distal artery. Imaging of the proximal left circumflex was performed using oblique sagittal planes passing from the origin of the artery at the division of the left main stem to the posterior atrioventricular groove. Perpendicular imaging planes were used at the site of signal loss for confirmation and to exclude vessel tortuosity.

\section{ANALYSIS OF MAGNETIC RESONANCE IMAGES}

All magnetic resonance images were reviewed in random order by two experienced observers (DJP, HGB) who were blinded to the results of $x$-ray angiography. Assessments were made in consensus. Contiguous image sets were reviewed individually and as a dynamic sequence. The images were first subjectively classified into three categories, according to quality for interpretation, as good, adequate or poor according to signal to noise, contrast to noise, and motion artefact. The vessel under interrogation was divided into thirds (proximal, mid and distal) and each was categorised visually as normal or showing significant signal loss. The degree of signal loss was classified as severe or partial, and in addition an irregular vessel outline was noted when present. The length of the signal loss, together with its distance from the arterial origin, was measured using on screen cursors. The proportional magnetic resonance signal loss was calculated by drawing a profile along the vessel and dividing the lowest signal value at the centre of loss by the maximum signal in the adjacent vessel.

\section{ANALYSIS OF CORONARY ANGIOGRAMS}

The $x$-ray angiograms were reviewed in random order by two experienced observers (DJP, HGB) who were blinded to the results of magnetic resonance angiography. Assessments were made in consensus. The location and percent luminal narrowing of any significant coronary arterial abnormality were assessed with callipers. Measurements were also made of the length of stenosis and the distance from the arterial origin. Absolute measurements were calculated from the known width of the 7 French gauge $(2.3 \mathrm{~mm}$ diameter) catheter on the film. A correction was applied for measurement of arterial lengths in the $x$-ray images, which would have been foreshortened by a projection angle not perpendicular to the artery. The actual angle of 
inclination of the artery was measured directly from the transaxial magnetic resonance images. When the arterial angle differed from the known projection angle, the foreshortening effect was corrected by simple geometry.

\section{STATISTICAL ANALYSIS}

The comparison of percentage stenosis in the three magnetic resonance groups (signal loss severe, partial or irregular wall only) was performed using analysis of variance. Intergroup differences were subsequently analysed using Scheffe's F-test. Comparison of magnetic resonance signal loss and percent diameter stenosis were performed using linear regression analysis. Comparisons between distances from the arterial origin and the lengths of the coronary stenoses by magnetic resonance imaging and $x$-ray coronary angiography were performed using a scatter plot and Bland-Altman analysis. ${ }^{8}$ Differences between the length of coronary stenosis between the two techniques in relation to the severity of the stenosis were analysed using Spearman's rank test. Twotailed tests were used where appropriate; a probability value of $<0.05$ was considered significant.

\section{Results}

DETECTION OF CORONARY STENOSIS

Image quality was considered good in 23 $(59 \%)$, adequate in $11(28 \%)$, and poor in five $(13 \%)$ cases. Forty seven $(85 \%)$ of 55 significant $(\geqslant 50 \%$ diameter reduction) coronary stenoses were identified by magnetic resonance imaging. Of 33 stenoses in the left anterior descending artery, 17 were in the proximal portion and $15(88 \%)$ of these were detected. The remaining 16 stenoses were in the midportion of the artery and $14(88 \%)$ were detected (fig 1). There were no stenoses in the distal portion. Of 17 stenoses in the right coronary artery, eight were in the proximal portion and six $(75 \%)$ were detected, while of eight stenoses in the mid-portion eight $(100 \%)$ were detected (fig 2). There were no stenoses in the distal portion of the artery, but one stenosis was present in the posterior descending artery, which was not detected. Three $(75 \%)$ of four stenoses in the proximal left circumflex artery were detected. There were no stenoses in the mid and distal portions. One left main stem stenosis was seen. Of the eight stenoses which were missed by magnetic resonance imaging, the stenosis severity was moderate $(64(12) \%$, range $50-80 \%$ ). In five cases, suboptimal quality images were a contributing factor, in two cases the tortuous path of the proximal right coronary artery hindered adequate imaging, and in one case the lesion was too distal and was not imaged at all.

In addition to the abnormalities shown by magnetic resonance imaging which were judged to be moderate or severe, there were three areas showing wall irregularity with minor luminal narrowing but no signal loss. At two of these sites, minor plaque formation was seen in the $x$-ray images with a luminal stenosis of $30 \%$, and at the third a $50 \%$ stenosis was
Figure 1 (A) Gradient echo image in a left oblique sagittal plane and $(B) x$ ray angiogram of a severe stenosis (arrow) in the mid-left anterior descending artery.
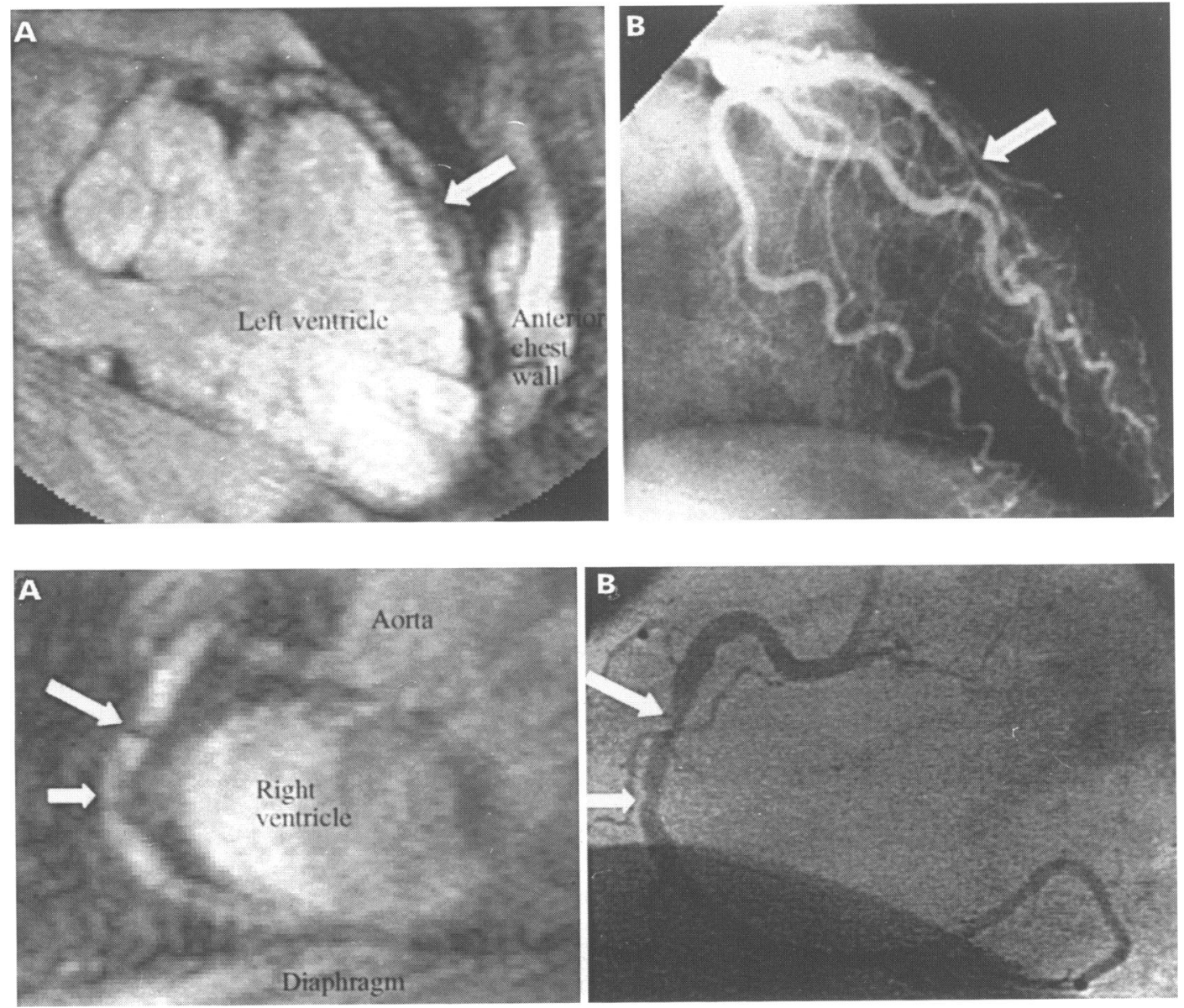

Figure 2 (A) Gradient echo image in a right oblique sagittal plane and (B) $x$-ray angiogram of two stenoses (arrows) in the proximal and mid-right coronary artery. 
Figure 3 (A) Scatter plot and (B) Bland-Altman plot comparing the distance to the stenosis as measured by $x$-ray and magnetic resonance angiography. The line of identity is shown. Overall agreement was good with increased scatter for more distal stenoses. MRI, magnetic resonance imaging. present. These wall irregularities were identified in magnetic resonance images of particularly high quality, but there were numerous other minor plaques in the $x$-ray images which were not detected by magnetic resonance.

There were five false positive areas of signal loss in the magnetic resonance images erroneously thought to represent significant coronary stenosis. In two cases this was caused by signal loss associated with passage of the artery in and out of the imaging plane (proximal left anterior descending artery and mid-right coronary artery). In retrospect the tortuosity could have been identified from the images obtained in the perpendicular plane. In two patients, an area of signal loss was ascribed to stenosis when the quality of the imaging was suboptimal (proximal right coronary artery), and in one patient signal loss was thought to represent a severe stenosis but was in fact localised artefact from a metal clip on an adjacent occluded right coronary saphenous bypass graft.

ASSESSMENT OF THE DISTANCE OF THE STENOSIS FROM THE ARTERIAL ORIGIN

Figure 3 shows the scatter plot and BlandAltman analysis of the distances of the stenoses identified by both techniques from
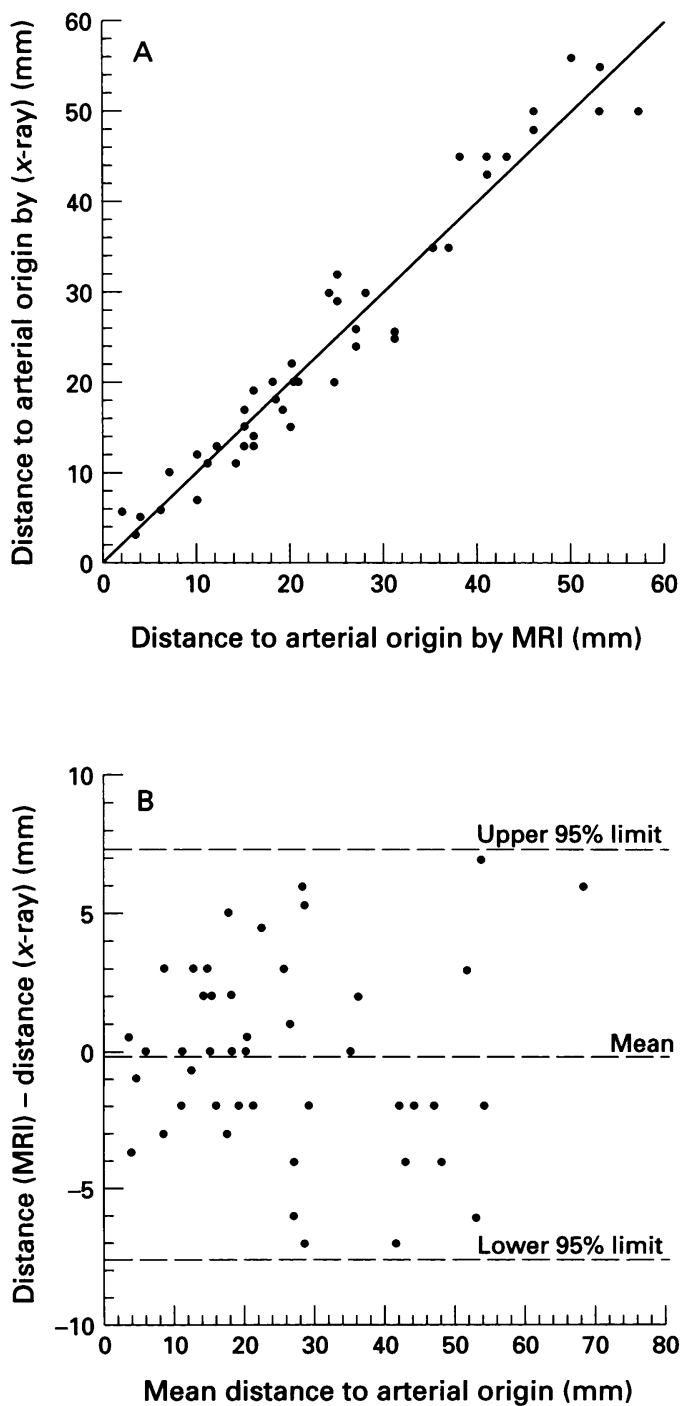
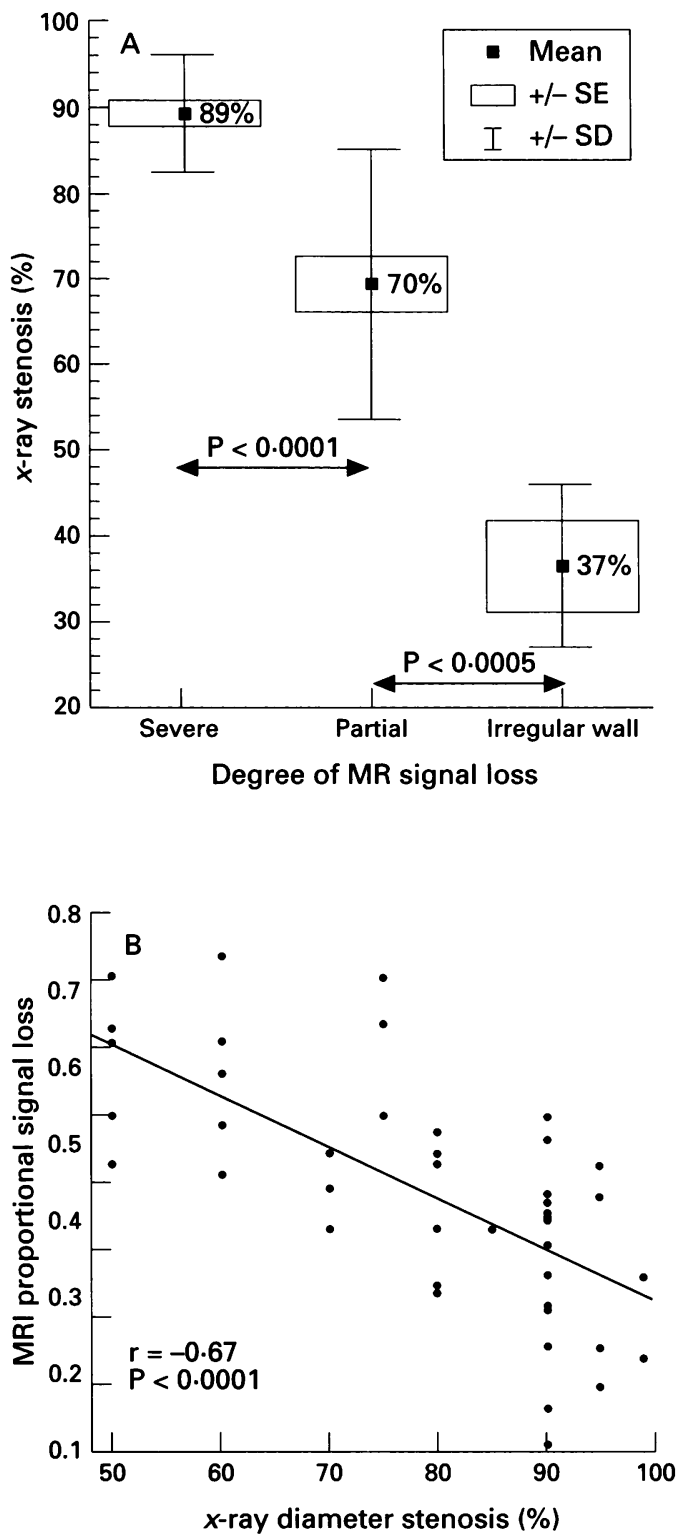

Figure 4 Comparison of severity of coronary stenosis as assessed by $x$-ray angiography and magnetic resonance imaging (MRI). (A) The mean percentage stenosis is significantly different in each group (severe/partial signal loss, or wall irregularity) suggesting that visual assessment of signal loss is a qualitative guide to stenosis severity. (B) The significant relation between the proportional magnetic resonance signal loss at the site of stenosis was measured directly on screen and the diameter stenosis severity was assessed by $x$-ray angiography. This confirms the qualitative results and shows the reasonably wide scatter that was observed. The line of regression is shown.

their arterial origin. There was close agreement between the techniques (magnetic resonance mean (SD) 27 (16) $\mathrm{mm}$ versus $x$-ray angiography $27(16) \mathrm{mm}, \mathrm{P}=\mathrm{NS}$, mean difference $-0.2 \mathrm{~mm}, 95 \%$ limits of agreement -7.7 to $7.2 \mathrm{~mm})$. The distance to 39 stenoses (83\%) agreed to within $5 \mathrm{~mm}$. The comparison of distances was particularly good for proximal stenoses within $25 \mathrm{~mm}$ of the origin, but the spread increased for more distal stenoses.

ASSESSMENT OF STENOSIS SEVERITY

ACCORDING TO SIGNAL LOSS

Comparison of the mean percent stenosis in the three groups showed significant variation 
Figure 5 (A) Scatter plot and (B) Bland-Altman analysis of the length of stenosis as assessed by magnetic resonance imaging (MRI) and $x$-ray angiography. There is more scatter than for the equivalent comparison of distance to stenosis with a net overestimation of stenosis length by MRI. The line of identity is shown.
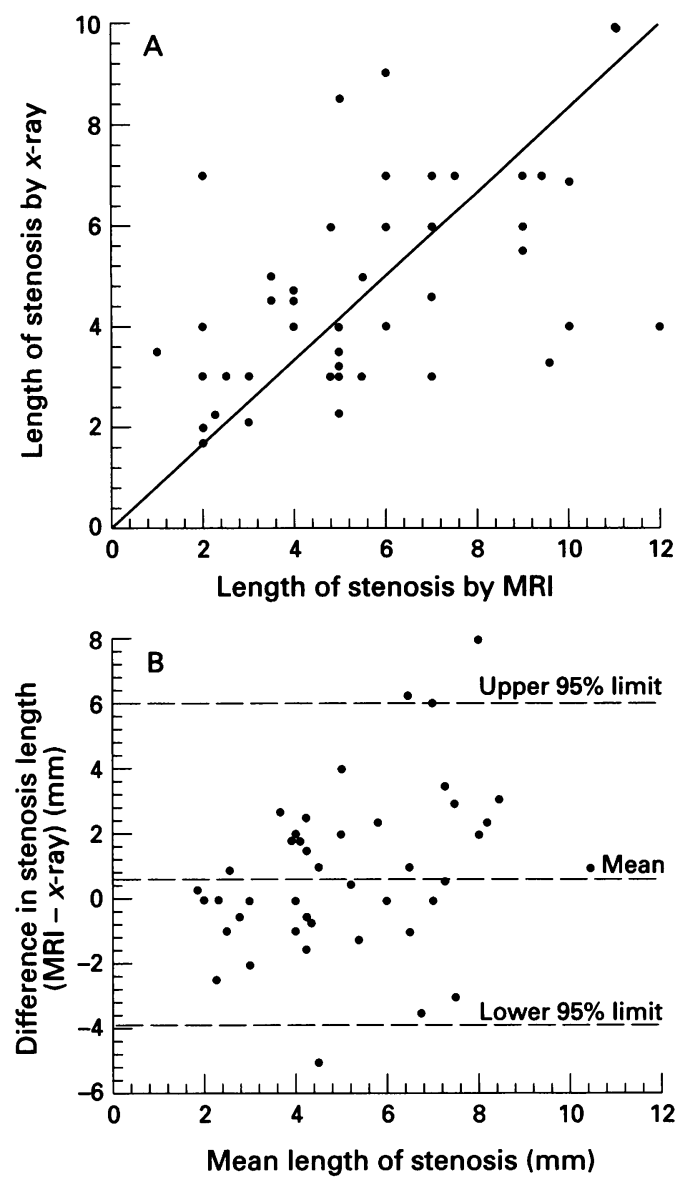

$(F=30 \quad \mathrm{P}<0.0001$, fig $4(\mathrm{~A}))$. The mean (SD) stenosis severity was $89(7) \%$, when severe signal loss was seen, which was significantly greater than the mean (SD) stenosis severity when partial signal loss (70 (16)\%) ( $P$ $<0.0001)$ or wall irregularity only was seen (37 (11)\%) $(P<0.00001)$. In addition, the difference between the partial signal loss and wall irregularity groups was also significant $(P<$ $0.0005)$. A further analysis of magnetic resonance signal loss was therefore performed using a profile analysis of magnetic resonance signal in the normal artery before and after the stenosis in comparison with the magnetic resonance signal at the stenosis. This was performed on screen and the proportional signal loss was calculated and compared with the percent diameter stenosis assessed by $x$-ray

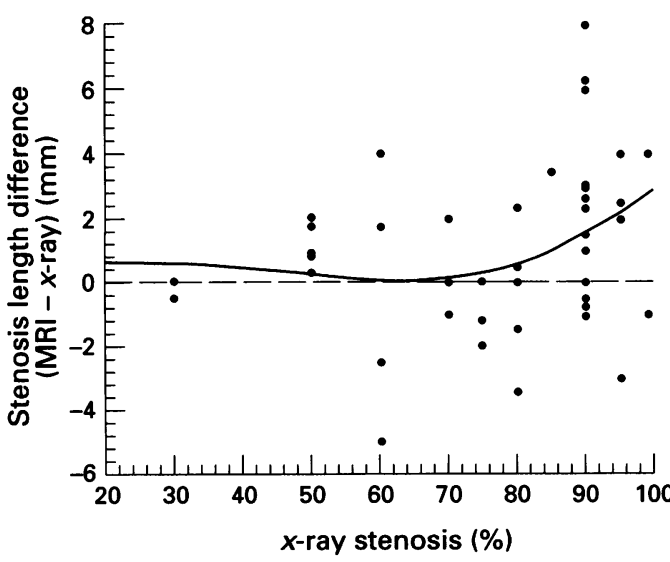

angiography. Figure 4 (B) shows a significant relation between stenosis severity and magnetic resonance signal loss $(r=-0.67 \mathrm{P}<$ 0.0001 ), although there is considerable scatter in the individual measurements.

\section{ASSESSMENT OF STENOSIS LENGTH}

Figure 5 shows the scatter plot and BlandAltman analysis of the length of the stenosis as assessed by magnetic resonance imaging and $x$-ray angiography. There was considerably more scatter than for the comparison of distances of stenoses from the arterial origin. Overall magnetic resonance imaging overestimated the length of coronary stenosis (6 (3) mm versus $x$-ray angiography $5(2) \mathrm{mm}, \mathrm{P}<$ 0.006 , mean difference $1.1 \mathrm{~mm}, 95 \%$ limits of agreement -4 to $6 \mathrm{~mm}$ ). A plot of the difference in stenosis length between magnetic resonance imaging and $x$-ray angiography with a least mean squares line fit suggested increasing overestimation by magnetic resonance as stenosis severity increased (fig 6). Spearman's rank test confirmed that this finding was significant $\left(r_{\mathrm{s}}=0.34, \mathrm{P}<0.02\right)$. Therefore, the higher stenosis length as assessed by magnetic resonance imaging occurred because of overestimation in arteries with the more severe stenoses.

\section{Discussion}

Magnetic resonance angiography of the larger and relatively stationary vessels has progressed rapidly over the past few years and the results now rival conventional angiography for carotid, ${ }^{9}$ renal, ${ }^{10}$ and peripheral arteries. ${ }^{11}$ Magnetic resonance imaging also has potential for non-invasive coronary artery imaging which is more difficult because of small size and rapid movement. In normal individuals, a combination of transaxial and oblique imaging has been shown to visualise $5 \mathrm{~cm}$ of the left anterior descending and right coronary arteries and rather less $(2.5 \mathrm{~cm})$ of the left circumflex artery, ${ }^{34}$ the difference being related to lower signal intensity in the posterior tissues because of the distance from the anterior placed surface coil. Experience with the technique is increasing, however, and in some patients up to $10 \mathrm{~cm}$ of the anteriorly located arteries and up to $6 \mathrm{~cm}$ of the posteriorly located artery may be seen. The new magnetic resonance technique needs to be validated against the $x$-ray method, and studies in normal individuals are supportive, with comparisons of the proximal coronary arterial diameters measured by magnetic resonance imaging not being statistically different from reference values, ${ }^{12}$ or the diameters measured by $x$-ray angiography. ${ }^{34}$ Comparisons of total coronary area and its relation to body surface area are also not statistically different from reference values. ${ }^{4}$ Resolution of the magnetic resonance technique at present is inferior, however, and there have been few studies in patients with coronary artery disease. ${ }^{5}$ It was the aim of this study to validate this new technique in assessment of coronary artery stenosis, including localisation, and the degree of severity and length of stenosis. 
Localising coronary artery stenosis by magnetic resonance imaging is important because it permits a confident comparison of the site of signal loss with the findings of $x$-ray angiography. Without an accurate comparison it is difficult to be certain that signal loss is in fact occurring at the site of stenosis, because of the differences in appearance of the tomographic and projection images. As shown in fig 3, there was a close correlation between the distances from the arterial origin to the stenosis by both techniques. In $83 \%$ of stenoses, the location measured by magnetic resonance imaging was within $5 \mathrm{~mm}$ of that measured by $x$-ray angiography. The agreement between the techniques was less good for the more distal lesions, but this probably reflects errors in measurements from the $x$-ray angiogram. In addition to showing that coronary stenosis can be accurately located by magnetic resonance imaging, it was usually also possible to establish the relation of the coronary stenosis to the nearest major branches, such as the septal and diagonal vessels for the left anterior descending artery, or the acute marginals for the right coronary artery. The branches of the left circumflex artery were less easy to identify with confidence. There are three implications of this result: firstly, that magnetic resonance signal loss is indeed occurring at the site of coronary stenosis; secondly, comparison of a tomographic angiogram with a projection angiogram may be made confidently for the localisation of stenosis; and thirdly, that when a suspicious area of signal loss is seen on the magnetic resonance angiogram, its location can be measured and used in subsequent images in oblique or perpendicular planes to establish whether the signal loss is constant and likely to be due to stenosis, or, for example, a tortuous curve.

The degree of stenosis is not accurately assessed by magnetic resonance angiography, but we have shown that qualitative variables can be used to divide stenoses broadly into moderate and severe according to the level of signal loss. Thus when severe signal loss was seen the mean diameter reduction was $89 \%$ compared with $70 \%$ when the signal loss was partial. A significant relation between the proportional magnetic resonance signal loss at the site of stenosis and percent diameter stenosis by $x$-ray angiography was shown to exist, although considerable variation was present in the results. Further work on defining this relation may improve results. Wall irregularities were also seen by magnetic resonance angiography, and these occurred at the site of plaques with minor luminal reduction by conventional criteria (mean 37\%). In addition, the length of the signal loss was also related to stenosis severity, such that magnetic resonance angiography overestimated the length progressively as the severity increased. Therefore both the intensity of the signal loss and its length may be useful in distinguishing severe from moderate stenosis. The reason for the increased signal loss with greater stenosis is probably related to turbulent flow which causes incoherent intravoxel phase dispersion and signal loss. This effect can be reduced by reducing the echo time of the sequence, but this may have an effect on the detection of less severe stenosis and requires higher gradient strengths.

\section{FUTURE DIRECTIONS}

The current resolution of magnetic resonance coronary angiography is lower than $x$-ray angiography, and it is unlikely therefore to allow a comparable estimation of diameter stenosis severity. Despite the well known problems with using percent diameter reduction as a standard for judging stenosis severity ${ }^{1314}$ it is nevertheless a commonly used clinical tool. One means by which magnetic resonance imaging might improve assessment of stenosis severity is to measure the increase in velocity at the stenosis, because the change is in proportion to the area of stenosis. This is achievable in principle using magnetic resonance velocity mapping to demonstrate the increase in velocity at the stenosis. Early studies of this technique in normal individuals have proved promising. ${ }^{15}$ In addition, magnetic resonance techniques for respiratory monitoring and feedback may help considerably by simplifying breath holding for the patient, allowing imaging in dyspnoeic patients and improving breath hold reproducibility. ${ }^{16}$ Faster imaging techniques are also likely to reduce coronary and respiratory motion artefact. ${ }^{17}$

\section{LIMITATIONS OF THIS STUDY}

This validation study was designed to compare magnetic resonance and $x$-ray angiography of individual stenoses and all patients were known to have coronary artery disease on the basis of thallium imaging or $x$-ray angiography. The patients therefore were highly selected. For this reason, no attempt has been made to quote specificity for detection in this study, and the sensitivity for detection of stenosis should be interpreted cautiously, because while we quote the results of detection, this was not the primary aim of this study and studies of patients with a lower incidence of coronary disease would yield different results. The single image breath hold technique used in this study has certain limitations which might limit its use clinically. The first problem is that of registration of different breath hold images despite efforts to encourage patients to hold their breath in a consistent way. The effect that this has is particularly noticeable in the transaxial images, but even in the oblique sagittal and coronal images translation or twisting of the artery may interfere with interpretation. A second problem is that of any inability to cooperate with the breath hold technique. This may occur because of inattention, or dyspnoea in the supine position from cardiac or respiratory problems. Technical advances as described earlier may alleviate these problems.

\section{Conclusions}

Significant technical and clinical progress is being made in magnetic resonance coronary angiography. This study demonstrates that in selected circumstances magnetic resonance with the current $2 \mathrm{D}$ breath hold technique is 
making encouraging progress towards the results of $x$-ray angiography for detection and localisation of stenosis, with a qualitative assessment of diameter stenosis severity and stenosis length. Widespread clinical application of magnetic resonance techniques will probably require faster image acquisition, reproducible breath hold techniques, and velocity measurements within the coronary arteries. Technical advances on all these fronts are in progress.

This study was funded in part by the British Heart Foundation and the Coronary Artery Disease Research Association. We thank the cardiologists at the Royal Brompton Hospital and Dr J M Walker at The Middlesex Hospital for permission to study their patients, and Picker International for their support.

1 Kennedy JW, Baxley WA, Bunnel IL, et al. Mortality related to cardiac catheterisation and angiography. Cather Cardiovasc Diagn 1982;8:323-40.

2 Davis K, Kennedy JW, Kemp HG, Judkins MP, Gosselin AJ, Killip T. Complications of coronary arteriography from the collaborative study of coronary artery surgery (CASS). Circulation 1979;59:1105-12.

3 Manning WJ, Li W, Boyle NG, Edelman RR. Fat-suppressed breath-hold magnetic resonance coronary pressed breath-hold magnetic reson

4 Pennell DJ, Keegan J, Firmin DN, Gatehouse PD, Underwood SR, Longmore DB. Magnetic resonance imaging of coronary arteries: technique and preliminary imaging of coronary arteries: technique and preliminary results. Br Hear $f$,

Manning WJ, Li W, Edelman RR. A preliminary report comparing magnetic resonance coronary angiography with conventional angiography. $N$ Engl f Med 1993;328: 828-32.

6 Duerinckx AJ, Urman MK. Two-dimensional coronary MR angiography: analysis of initial clinical results.
Radiology 1994;193:731-8.

7 Edelman RR, Wallner B, Singer A, Atkinson DJ, Saini S Segmented turboFLASH: method for breath hold MR imaging of the liver with flexible contrast. Radiology 1990;177:515-21.

8 Bland JM, Altman DG. Statistical method for assessing agreement between two methods of clinical measurement. Lancet 1986;i:307-10.

9 Fram EK, Heiserman JE. Carotid and vertebral arteries. In Potchen EJ, Haacke EM, Siebert JE, Gottschalk A, eds. Magnetic resonance angiography, concepts and applications. St Louis, USA: Mosby, 1993:498-518.

10 Kent KC, Edelman RR, Kim DD, Steinman TI, Porter $\mathrm{DH}$, Skillman JJ. Magnetic resonance imaging: a reliable test for the evaluation of proximal atherosclerotic renal arterial stenosis. $¥$ Vasc Surg 1991;13:311-8.

11 Owens RS, Carpenter JP, Baum RA, Perloff LJ, Cope C. Magnetic resonance imaging of angiographically occult Magnetic resonance imaging of angiographically occult runoff vessels in peripheral ar

12 Dodge JT, Brown BG, Bolson EL, Dodge HT. Lumen diameter of normal coronary arteries. Influence of age, sex, anatomic variation, and left ventricular hypertrophy or dilatation. Circulation 1992;86:232-46.

13 Kleiman NS, Rodriguez AR, Raizner AE. Interobserve variability in grading of coronary arterial narrowing using the American College of Cardiology/American Heart Association grading criteria. Am 7 Cardiol 1992; 69:413-5.

14 White CW, Wright CB, Doty DB, Hiratza LF, Eastham $\mathrm{CL}$, Harrison DG, et al. Does visual interpretation of the coronary arteriogram predict the physiologic importance of a coronary stenosis? N Engl f Med 1984;310:819-24.

15 Keegan J, Firmin D, Gatehouse P, Longmore D. The application of breath-hold phase velocity mapping techvelocity: phantom data and initial in vivo results. Magn Reson Med 1994;31:1-11.

16 Liu YL, Riederer SJ, Rossman PJ, Grimm RC, Debbins JP, Ehman RL. A monitoring, feedback, and triggering system for reproducible breath-hold MR imaging. Magn Reson Med 1993;30:507-11.

17 Meyer CH, Hu BS, Nishimura DG, Macovski A. Fast spiral coronary artery imaging. Magn Reson Med 1992;28: 202-13. 